\title{
Rotating solitary wave at the wall of a cylindrical container
}

\author{
Mustapha Amaouche \\ Department de Physique, Université de Béjaia, Béjaia, Algeria \\ Hamid Ait Abderrahmane* \\ Mathematical and Computer Sciences and Engineering Division, King Abdullah University of Science and Technology (KAUST), \\ Thuwal, Saudi Arabia \\ Georgios H. Vatistas \\ Department of Mechanical and Industrial Engineering, Concordia University, Montréal, Québec, Canada H3G $1 M 8$
}

(Received 30 July 2012; revised manuscript received 9 March 2013; published 30 April 2013)

\begin{abstract}
This paper deals with the theoretical modeling of a rotating solitary surface wave that was observed during water drainage from a cylindrical reservoir, when shallow water conditions were reached. It represents an improvement of our previous study, where the radial flow perturbation was neglected. This assumption led to the classical planar Korteweg-de Vries equation for the wall wave profile, which did not account for the rotational character of the base flow. The present formulation is based on a less restricting condition and consequently corrects the last shortcoming. Now the influence of the background flow appears in the wave characteristics. The theory provides a better physical depiction of the unique experiment by predicting fairly well the wave profile at least in the first half of its lifetime and estimating the speed of the observed wave with good accuracy.
\end{abstract}

DOI: $10.1103 /$ PhysRevE.87.043015

PACS number(s): 47.35.Fg

\section{INTRODUCTION}

Solitary waves are localized single elevations or depressions [1]. Due to the balance between the nonlinearity and dispersion these propagate without any appreciable temporal changes in their shapes and speeds. Similar waves have been observed in various fields of physics such as, for example, hydrodynamics, acoustics, plasma, and optics [2].

The presence of a background rotation significantly influences the propagation of both surface and internal gravity solitary waves. For instance, in the case of internal waves, developed in a shallow rotating channel, the degree of influence of the rotation depends on its strength [3]. When the rotation is intense, its effect is distinguishable from those of weak nonlinearity and dispersion and results in a transverse exponential decay of the solitary wave's amplitude. However, when the rotation is of the same order of magnitude as the nonlinearity and dispersion, the internal gravity waves are described by the rotation-modified Kadomstev-Petriashvili (KP) equation rather than by the classical Korteweg-de Vries (KdV) equation [3-5]. At the limit of infinitely large channel width, the KP equation reduces to the Ostrovsky equation, which was first derived to account for the effects of the earth's rotation on oceanic internal solitary waves [6].

A background rotation can also induce inertial solitary waves, as in the case of liquid-filled upright cylindrical containers [7]. In this case, the wave is axisymmetric and propagates back and forth along the vortex core between the liquid free surface and the bottom of the container. Similar inertial solitary waves, moving along inside the vortex cores, were also observed within rotating turbulent flows [8,9]. Cases where solitary waves have been observed in swirling flows are very few. They are limited to the configurations where the vortex core serves as the waveguide along which they propagate.

\footnotetext{
*haitabd@hotmail.com
}

This paper deals with yet another type of solitary wave in rotating flows, observed during liquid drainage through the bottom port of a vertical cylindrical container, whereby a shallow layer of fluid inside the cylindrical tank is in a state of rotation around the vertical axis. When the solitary wave appears, it begins to gyrate in the azimuthal direction. This event was parenthetically reported by Vatistas [10] and recently modeled by the evolutionary equation of Korteweg and de Vries [11]. To the best of our knowledge, there are no documented cases of similar rotating solitary waves observed in nature or technology. For the moment, the only available accounts of this distinctive phenomenon are the laboratory observations reported previously $[10,11]$ and those described in the present article. However, we trust that the present experimental apparatus might be improved to allow investigations, under more controllable conditions, that will determine the role of a background rotation in the propagation of solitary waves. The last general theme is currently receiving much attention, particularly the case of internal solitary waves $[12,13]$. In fact, the role of the earth's rotation in the propagation of internal solitary waves in oceans remains not fully understood. It is worth noting that recently an experimental setup, somewhat akin to the one used in our experiments, was considered as a shallow water analog to describe the physics of a stellar core and its associated standing accretion shock instability [14].

\section{GOVERNING EQUATIONS}

The classical KdV equation arises from the conservation equations for irrotational motion of an incompressible inviscid fluid bounded above by a free surface and below by a rigid horizontal plane. For the case under consideration, the wave motion emerges from a primary rotating flow in which a swirl velocity $r \Omega_{0}$ is imparted; this yields a certain radial distribution of the fluid layer thickness. The resulting perturbed motion is rotational and accordingly it must be described by the full 
Euler equations. In contrast to the basic flow, the perturbed velocity is three dimensional and the governing equations in dimensionless form are

$$
\begin{gathered}
v_{\theta}+(r u)_{r}+r w_{z}=0 \\
D_{t} u-2 \Omega v+\varepsilon\left(u u_{r}+\frac{1}{r} v u_{\theta}-\frac{v^{2}}{r}+w u_{z}\right)+p_{r}=0 \\
D_{t} v+2 \Omega u+\varepsilon\left(u v_{r}+\frac{1}{r} v v_{\theta}+\frac{u v}{r}+w v_{z}\right)+\frac{1}{r} p_{\theta}=0 \\
\delta\left[D_{t} w+\varepsilon\left(u w_{r}+\frac{1}{r} v w_{\theta}+w w_{z}\right)\right]+p_{z}=0
\end{gathered}
$$

with the boundary conditions

$$
\begin{gathered}
\left.w\right|_{z=0}=0, \\
\left.u\right|_{r=1}=0, \\
p_{0}+\left.\varepsilon p\right|_{z=h_{0}+\varepsilon \eta}=0, \\
\left.w\right|_{z=h_{0}+\varepsilon \eta}=D_{t} \eta+\varepsilon\left(u \eta_{r}+\frac{1}{r} v \eta_{\theta}\right) .
\end{gathered}
$$

In Eqs. (1)-(8), written in cylindrical coordinates $(r, \theta, z)$, the subscripts indicate differentiation with respect to the corresponding variables, $u, v$, and $w$ are the radial, azimuthal, and axial velocity components, respectively, $p$ is the pressure perturbation, and $\eta$ indicates the free surface elevation. The parameters appearing in Eqs. (1)-(4) are $\varepsilon=\frac{A}{H_{0}}$ and $\delta=\frac{H_{0}^{2}}{R^{2}}$, where $A$ is a measure of the wave amplitude, $R$ is the cylinder radius which serves to scale horizontal distances, and $H_{0}$ is the undisturbed fluid depth at the lateral wall, considered here as the typical length scale in the axial direction. The velocity components $u$ and $v$ are in units of $\varepsilon \sqrt{g H_{0}}$, and $w$, time, and pressure are scaled by $\varepsilon \sqrt{g H_{0} \delta}, R / \sqrt{g H_{0}}$, and $\rho g H_{0}$, respectively. The symbol $D_{t}$ stands for the total time derivative, $\partial_{t}+\Omega \partial_{\theta}$ where $\Omega$ denotes the relative background rotation in comparison with $\sqrt{g H_{0}} / R, h_{0}=1+\frac{1}{2} \Omega^{2}\left(r^{2}-1\right)$ is the dimensionless fluid depth, and $p_{0}=h_{0}-z$ is the basic pressure distribution. As in the planar case, we are interested in small amplitude waves, i.e., in the limits as $\varepsilon \rightarrow 0$ and $\delta \rightarrow 0$. It turns out that one choice which leads to a solitary wave profile for $\eta$ is to set $\delta=O(\varepsilon)$ as $\varepsilon \rightarrow 0$. To carry on the analysis, we suppose that the solution to Eqs. (1)-(8) exists in the form of a power series in $\varepsilon$, namely, $f=f_{0}+\varepsilon f_{1}+O\left(\varepsilon^{2}\right)$ for any dependent variable $f$.

\section{PRELIMINARY RESULTS}

The continuity equation along with the impermeability condition at the bottom give

$$
w=-\frac{1}{r} \int_{0}^{z}\left[v_{\theta}+(r u)_{r}\right] d \xi
$$

were $\xi$ indicates the vertical integration variable. Integrating the axial momentum equation with respect to $z$ and using the free surface boundary condition for the pressure lead to

$$
p=\eta+\delta \Psi,
$$

where

$$
\Psi=\frac{1}{r} \int_{h_{0}}^{z} d \nu \int_{0}^{v} D_{t}\left[v_{0 \theta}+\left(r u_{0}\right)_{r}\right] d \xi
$$

is the dispersive part of the disturbed pressure. Then, rewriting the azimuthal and radial momentum equations with the above expression for $p$ and neglecting terms of order $\varepsilon^{2}, \delta \varepsilon$, and higher, one obtains, respectively,

$$
\begin{aligned}
& D_{t} v+2 \Omega u+\varepsilon\left(u_{0} v_{0 r}+\frac{1}{r} v_{0} v_{0 \theta}+\frac{u_{0} v_{0}}{r}+w_{0} v_{0 z}\right) \\
& +\frac{1}{r}\left(\eta_{\theta}+\delta \Psi_{0 \theta}\right)=0, \\
& D_{t} u-2 \Omega v+\varepsilon\left(u_{0} u_{0 r}+\frac{1}{r} v_{0} u_{0 \theta}-\frac{v_{0}^{2}}{r}+w_{0} u_{0 z}\right) \\
& \quad+\eta_{r}+\delta \Psi_{0 r}=0 .
\end{aligned}
$$

These two equations are associated with the free surface kinematic equation, which becomes

$$
D_{t} \eta+\varepsilon\left(u_{0} \eta_{r}+\frac{1}{r} v_{0} \eta_{\theta}\right)+\frac{1}{r} \int_{0}^{h_{0}+\varepsilon \eta}\left[v_{\theta}+(r u)_{r}\right] d z=0 .
$$

Before handling the full nonlinear eigenvalue problem, let us first consider the leading order problem, which reads

$$
\begin{gathered}
D_{t} v+2 \Omega u+\frac{1}{r} \eta_{\theta}=0, \\
D_{t} u-2 \Omega v+\eta_{r}=0 .
\end{gathered}
$$

These equations imply that, at zeroth order in $\varepsilon$ and $\delta, u$ and $v$ do not depend on the axial coordinate. So the free surface kinematic condition takes the form

$$
D_{t} \eta+\frac{h_{0}}{r}\left[v_{\theta}+(r u)_{r}\right]=0 .
$$

A complete solution of Eqs. (15)-(17) can be obtained in the form of a traveling wave in the azimuthal direction. The distribution of the modal wave amplitude is then described by an equation similar to the Bessel equation. It differs from the latter only in the presence of the basic depth $h_{0}(r)$ (see the Appendix for the derivation). In the present analysis, we will restrict our consideration to only an approximate description of the wave motion at the wall where $h_{0}=1$.

As a matter of fact, the elliptic character of the flow in the radial direction does not allow an exact description of the wall motion without some additional approximation at the wall instead of the condition prescribed by the axial symmetry of the problem. Neglecting the radial flow leads to a planar KdV equation,which is incapable of accounting for the rotational character of the flow [11]. In order to correct this defect, we make here a less stringent approximation, which consists of neglecting the radial curvature of the radial velocity at the wall. It is to be noted that by requiring the cancellation of any higher order radial derivative of the radial flow one can perform a similar analysis. However, the result would be just a slightly better approximation after much more tedious calculations. 
Hence, by making use of this approximation $\left(u_{r r}^{*}=0\right)$ and performing a Taylor expansion, up to first order in $\delta r$ near $r=1,(15)-(17)$ yield

$$
\begin{gathered}
D_{t} v^{*}+\eta_{\theta}^{*}=0, \\
\eta_{r}^{*}-2 \Omega v^{*}=0, \\
D_{t} \eta^{*}+v_{\theta}^{*}+u_{r}^{*}=0, \\
D_{t}\left(v^{*}+v_{r}^{*}\right)+2 \Omega u_{r}^{*}+\eta_{r \theta}^{*}=0, \\
D_{t}\left(\eta^{*}+\eta_{r}^{*}\right)+\Omega^{2}\left(v_{\theta}^{*}+u_{r}^{*}\right)+v_{r \theta}^{*}+2 u_{r}^{*}=0, \\
D_{t} u_{r}^{*}-2 \Omega v_{r}^{*}+\eta_{r r}^{*}=0 .
\end{gathered}
$$

In these equations the asterisk indicates the wall-valued variables. We observe that the term $u_{r r}^{*}$ is dropped in Eq. (22) owing to our assumption. We seek a solution to the eigenvalue problem (18)-(22) in the traveling wave form in the azimuthal direction, i.e., depending on the variable $X=\theta-\left(\Omega+\frac{1}{\alpha}\right) t$, $\alpha$ being an eigenvalue to be determined. This amounts to setting $D_{t}=-\frac{1}{\alpha} \partial_{\theta}+O(\varepsilon, \delta)$. So a nontrivial solution for $\left(\eta^{*}, \eta_{r}^{*}, v^{*}, v_{r}^{*}, u_{r}^{*}\right)$ exists provided that $\alpha$ satisfies the eigenvalue relation

$$
3 \alpha^{2}=\Omega^{2}+1 .
$$

The corresponding eigenvector then follows as

$$
\begin{gathered}
v^{*}=\alpha \eta^{*}+O(\varepsilon, \delta), \\
\eta_{r}^{*}=2 \Omega \alpha \eta^{*}+O(\varepsilon, \delta), \\
v_{r}^{*}=(2 \Omega-\alpha) \eta^{*}+O(\varepsilon, \delta), \\
u_{r}^{*}=\left(\frac{1}{\alpha}-\alpha\right) \eta_{\theta}^{*}+O(\varepsilon, \delta),
\end{gathered}
$$

where $\eta^{*}$ remains an arbitary function of $r$ and $X$. We observe that the relation (27) can also be expressed as $v_{r}^{*}=$ $\left(2 \frac{\Omega}{\alpha}-1\right) v^{*}+O(\varepsilon, \delta)$. Experiments show that the free surface elevation $\eta$ increases in the radial direction. This implies, owing to Eq. (26), that only the positive value of $\alpha$ must be retained, as in the planar case where $\alpha=1$. It is to be noted that (23) is added to the previous system only to show that $\eta_{r r}^{*}$ and $u_{r r}^{*}$ cannot be neglected simultaneously. In the present case, $\eta_{r r}^{*}$ falls, if desired, as a result of the eigenvalue problem.

\section{NONLINEAR AND DISPERSIVE CORRECTIONS}

\section{A. Pressure distributuion}

Before proceeding any further, we first express the pressure $\Psi$ and its horizontal gradient. Since $v_{0}$ and $u_{0}$ do not depend on $z$, the pressure takes the form

$$
\Psi=\frac{1}{2 r}\left(z^{2}-h_{0}^{2}\right) D_{t}\left[v_{0 \theta}+\left(r u_{0}\right)_{r}\right]+O(\epsilon, \delta) .
$$

With Eq. (17) in mind, one can rewrite $\Psi$ as

$$
\Psi=\frac{1}{2 h_{0}}\left(h_{0}^{2}-z^{2}\right) D_{t}^{2} \eta+O(\epsilon, \delta),
$$

which, owing to (24), reduces at $r=1$ to

$$
\Psi^{*}=\frac{1}{2 \alpha^{2}}\left(1-z^{2}\right) \eta_{\theta \theta}^{*}+O(\varepsilon, \delta)
$$

While the azimuthal gradient of $\Psi$ at $r=1$ may be expressed directly from Eq. (31) as

$$
\Psi_{\theta}^{*}=\frac{1}{2 \alpha^{2}}\left(1-z^{2}\right) \eta_{\theta \theta \theta}^{*}+O(\varepsilon, \delta),
$$

its radial gradient must be deduced from Eq. (30), after some elementary algebra,

$$
\Psi_{r}=\frac{1}{2} D_{t}^{2}\left\{h_{0 r}\left(1+\frac{z^{2}}{h_{0}^{2}}\right) \eta+\left(h_{0}-\frac{z^{2}}{h_{0}}\right) \eta_{r}\right\},
$$

which, by making use of Eqs. (25) and (26), yields at $r=1$

$$
\Psi_{r}^{*}=\frac{\Omega}{\alpha}\left\{1+\frac{\Omega}{2 \alpha}+\left(\frac{\Omega}{2 \alpha}-1\right) z^{2}\right\} \eta_{\theta \theta}^{*}+O(\varepsilon, \delta) .
$$

\section{B. Azimuthal equation and kinematic free surface condition}

Nonlinearity and dispersion will be included in these equations by seeking $u$ and $v$ as expansions of the form

$(u, v)=\left(u_{0}, v_{0}\right)+\varepsilon\left(u_{1}, v_{1}\right)+\delta\left(u_{2}, v_{2}\right)+O\left(\varepsilon^{2}, \delta^{2}, \varepsilon \delta\right)$,

where $v_{0}^{*}=\alpha \eta^{*}$ and $u_{0 r}^{*}=\left(\frac{1}{\alpha}-\alpha\right) \eta_{\theta}^{*} ;\left(u_{1}, v_{1}\right)$ and $\left(u_{2}, v_{2}\right)$ are nonlinear and dispersive corrections whose wall values will be defined afterwards. Therefore, the projection on the lateral wall of the azimuthal momentum equation is

$$
\alpha D_{t} \eta^{*}+\eta_{\theta}^{*}+\varepsilon\left(\alpha^{2} \eta^{*} \eta_{\theta}^{*}+D_{t} v_{1}^{*}\right)+\delta\left(D_{t} v_{2}^{*}+\Psi_{\theta}^{*}\right)=0 .
$$

On integrating through the fluid depth with Eqs. (25) and (32) in mind, (36) yields

$$
\begin{gathered}
\alpha D_{t} \eta^{*}+\eta_{\theta}^{*}+\varepsilon\left(\alpha^{2} \eta^{*} \eta_{\theta}^{*}-\frac{1}{\alpha} V_{1 \theta}\right) \\
+\delta\left(\frac{1}{3 \alpha^{2}} \eta_{\theta \theta \theta}^{*}-\frac{1}{\alpha} V_{2 \theta}\right)=0,
\end{gathered}
$$

where $V_{1}$ and $V_{2}$ are the depth-averaged values of $v_{1}^{*}$ and $v_{2}^{*}$ that will be determined later on by invoking the compatibility of Eq. (37) with Eq. (14). The latter is first transformed by expressing the integrand $v_{\theta}+(r u)_{r}$ as follows. Multiplying (12) by $r$, differentiating it with respect to $r$, and then projecting it on $r=1$, by equating terms of the same order in $\varepsilon$ and $\delta$, we get

$$
\begin{aligned}
(r u)_{r}^{*}= & \left(r u_{0}\right)_{r}^{*}-\frac{1}{2 \Omega}\left\{\varepsilon\left[D_{t}\left(r v_{1}\right)_{r}^{*}+N_{r}^{*}\right]\right. \\
& \left.+\delta\left[D_{t}\left(r v_{2}\right)_{r}^{*}+\Psi_{\theta r}^{*}\right]\right\}
\end{aligned}
$$

with

$$
\begin{aligned}
N_{r}^{*} & =\left(v_{0} v_{0 \theta}+r u_{0} v_{0 r}+u_{0} v_{0}\right)_{r}^{*} \\
& =2\left(\Omega \alpha+\frac{\Omega}{\alpha}-\alpha^{2}\right) \eta^{*} \eta_{\theta}^{*} .
\end{aligned}
$$

Then,

$$
\begin{aligned}
(r u)_{r}^{*}+v_{\theta}^{*}= & v_{0 \theta}^{*}+\left(r u_{0}\right)_{r}^{*}+\varepsilon\left\{v_{1 \theta}^{*}-\frac{1}{2 \Omega}\left[D_{t}\left(r v_{1}\right)_{r}^{*}+N_{r}^{*}\right]\right\} \\
& +\delta\left\{v_{2 \theta}^{*}-\frac{1}{2 \Omega}\left[D_{t}\left(r v_{2}\right)_{r}^{*}+\Psi_{\theta r}^{*}\right]\right\} .
\end{aligned}
$$


Integrating through the fluid depth and taking the zeroth order results into account gives

$$
\begin{aligned}
& \int_{0}^{1+\varepsilon \eta^{*}}\left[(r u)_{r}^{*}+v_{\theta}^{*}\right] d z \\
& =\frac{1}{\alpha} \eta_{\theta}^{*}+\varepsilon\left\{\left(1+\frac{1}{\alpha^{2}}\right) V_{1 \theta}+\alpha\left(\frac{\alpha}{\Omega}-1\right) \eta^{*} \eta_{\theta}^{*}\right\} \\
& \quad+\delta\left\{\left(1+\frac{1}{\alpha^{2}}\right) V_{2 \theta}-\frac{1+\Omega}{3 \alpha} \eta_{\theta \theta \theta}^{*}\right\} .
\end{aligned}
$$

Hence, the projection on $r=1$ of Eq. (14) takes the form

$$
\begin{aligned}
& \alpha D_{t} \eta^{*}+\eta_{\theta}^{*}+\varepsilon\left\{\left(\alpha+\frac{1}{\alpha}\right) V_{1 \theta}+\alpha^{2}\left(\frac{\alpha}{\Omega}-1\right) \eta^{*} \eta_{\theta}^{*}\right\} \\
& +\delta\left\{\left(\alpha+\frac{1}{\alpha}\right) V_{2 \theta}-\frac{1+\Omega}{3} \eta_{\theta \theta \theta}^{*}\right\}=0 .
\end{aligned}
$$

Equations (37) and (41) are compatible provided that $V_{1 \theta}=\frac{\alpha^{3}(2 \Omega-\alpha)}{\Omega\left(\alpha^{2}+2\right)} \eta^{*} \eta_{\theta}^{*}$ and $V_{2 \theta}=\frac{1+\alpha^{2}(1+\Omega)}{3 \alpha\left(2+\alpha^{2}\right)} \eta_{\theta \theta \theta}^{*}$. The free surface elevation is finally described by the following KdV-type equation:

$$
\eta_{t}^{*}+\left(\Omega+\frac{1}{\alpha}\right) \eta_{\theta}^{*}+\varepsilon E(\alpha, \Omega) \eta^{*} \eta_{\theta}^{*}+\delta D(\alpha, \Omega) \eta_{\theta \theta \theta}^{*}=0
$$

with $E(\alpha, \Omega)=\frac{\alpha^{3}+\Omega\left(2-\alpha^{2}\right)}{\Omega\left(2+\alpha^{2}\right)} \alpha^{2}$ and $D(\alpha, \Omega)=\frac{1-\Omega \alpha^{2}}{3 \alpha^{2}\left(2+\alpha^{2}\right)}$.

The solution of Eq. (42) is

$$
\eta^{*}(\theta, t)=\operatorname{sech}^{2} \frac{1}{2}\left(\frac{\varepsilon E}{3 \delta D}\right)^{1 / 2}\left\{\theta-\left(\Omega+\frac{1}{\alpha}+\frac{1}{3} \varepsilon E\right) t\right\} .
$$

In dimensional form, the wall free surface elevation is given by

$$
\begin{aligned}
\eta^{*}(\theta, t)= & A \operatorname{sech}^{2} \frac{1}{2 H_{0}}\left(\frac{E_{0}}{3 D_{0}}\right)^{1 / 2} \\
& \times\left\{R \theta-\left(R \Omega_{0}+\frac{\sqrt{g H_{0}}}{\alpha_{0}}+\frac{A \sqrt{g H_{0}}}{3 H_{0}} E_{0}\right) t\right\},
\end{aligned}
$$

where $E_{0}, D_{0}$, and $\alpha_{0}$ indicate the expressions of $E, D$, and $\alpha$ once $\Omega$ is replaced by $\Omega_{0} R / \sqrt{g H_{0}}$.

\section{EXPERIMENT}

\section{A. Experimental setup}

The theoretical wave profile (44) is compared to the experimentally observed one in a Plexiglas cylindrical container of diameter $D$ equal to $285 \mathrm{~mm}$. The drainage hole of diameter $(d)$ equal to $31.7 \mathrm{~mm}$ was located at the center of the container's lower plate. The tank was filled with water. Two initial water heights $h=435$ and $h=270 \mathrm{~mm}$ were considered. Note that the initial height was found to have no influence on the solitary wave of interest here. For the purpose of visualization, a blue water-soluble dye (food coloring) was added to the fluid prior to the experiments.

The experimental setup is shown in Fig. 1(a). The water within the tank was stirred with a rod, in a way similar to that of Pritchard [7], and soon afterwards the rod was lifted and the bottom manifold was opened for the water to drain. As the water level approached the shallow water condition the amplitude of the free surface oscillations increased and matured into a single surface bulge (solitary wave). At this time, we replugged the exit, and thus the water depth remained constant. The solitary wave revolved around the cylindrical wall for several laps (approximately 30) before it vanished. It is worth noting that the solitary wave could be observed only when enough initial vorticity was present prior to the commencement of the draining process. This can be viewed either as the residual vorticity due to the reservoir filling process, or as swirl introduced by stirring the water. In fact, if the tank is filled and then left undisturbed for a while to calm down prior to draining (i.e., there is almost no residual vorticity), the solitary wave may not appear at all. The event was recorded using a CCD camera and a sample of the revolving solitary wave is shown in Fig. 1(b). The wave profile was detected using a digital image processing technique. The temporal free surface elevation, in reference to the bottom of the container was obtained at a fixed angular location with respect to the tank cross section. For convenience, the angular
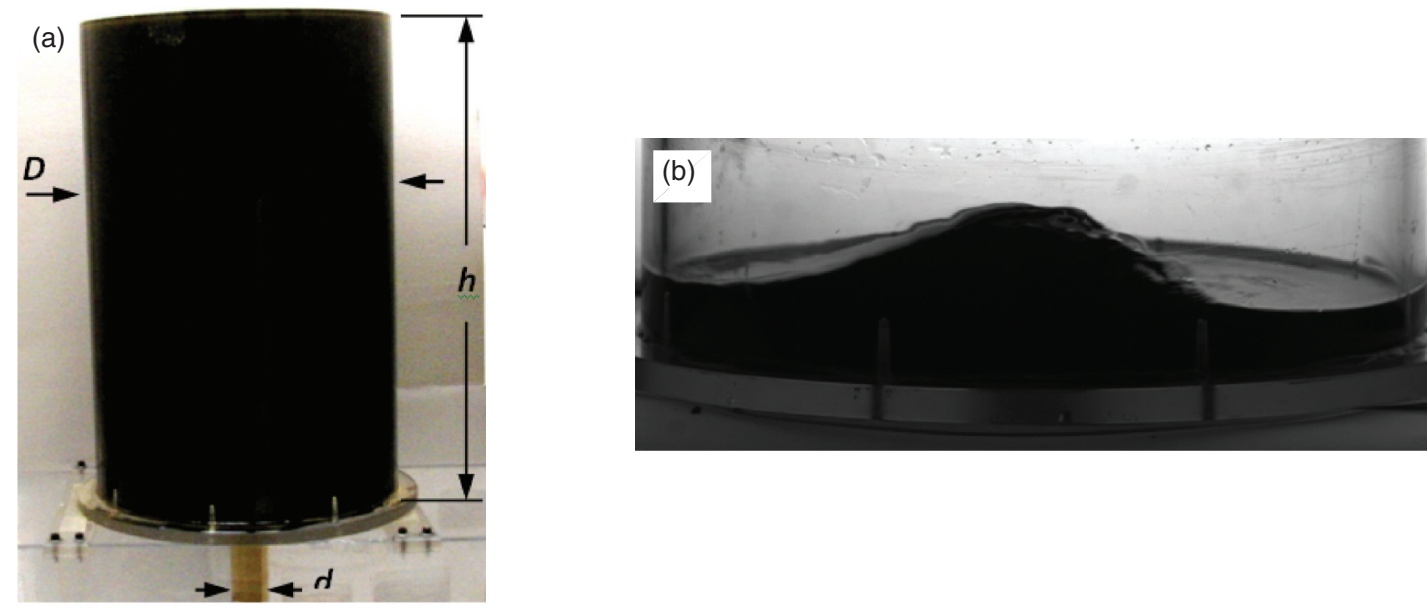

FIG. 1. (Color online) (a) Experimental setup. Plexiglas cylindrical reservoir with a central drainage hole located at the bottom center of the container. (b) A typical solitary wave. 

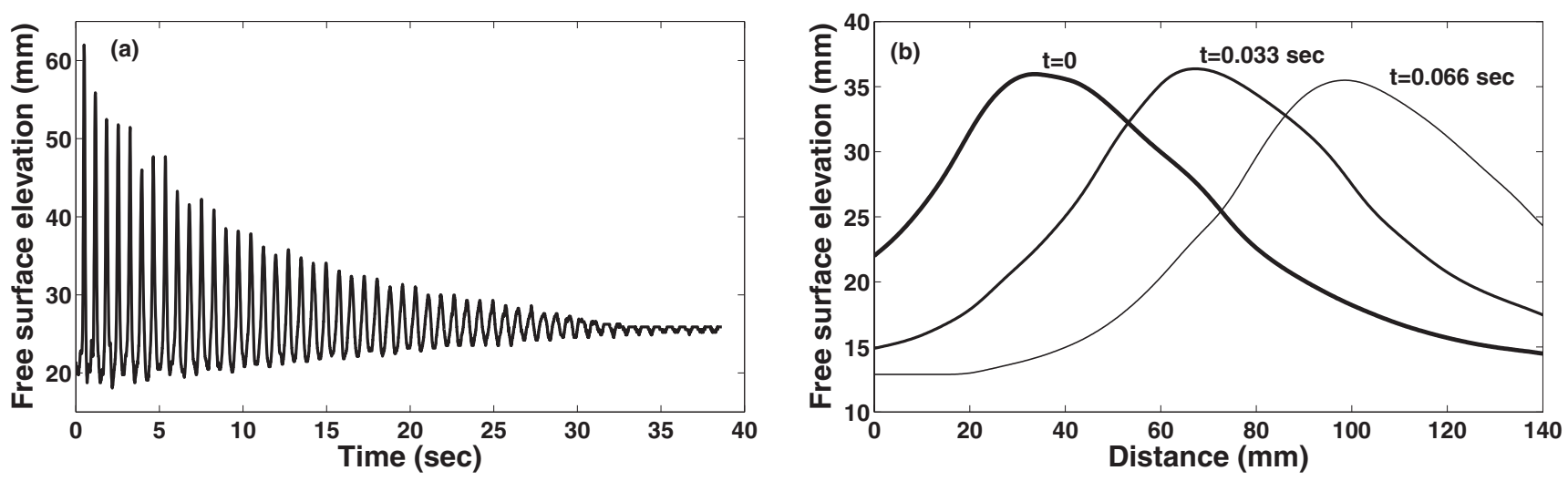

FIG. 2. Free surface elevation (a) recorded at a given location considered as $\theta=0$; (b) in three snapshots of the wave at three successive times.

position that corresponded to the middle section of the images, Fig. 1(b), was selected to be the origin $(\theta=0)$. An example of the free surface elevation is given in Fig. 2.

\section{B. Results and discussion}

In the analysis above, the rotational speed of the background flow, $\Omega_{0}$, is a fundamental parameter to which we did not have direct access from the experiments. This speed was estimated through the measurement of the wave speed in the laboratory frame of reference, which is the sum of the intrinsic wave speed in the fluid frame of reference and the rotational speed of the carrier fluid, $\Omega_{0}$. The wave speed in the laboratory frame of reference could be calculated in two ways. The first way used the periods of the free surface oscillations shown in Fig. 2(a), which is in fact the time between two subsequent laps of the solitary wave. The second method utilizes two subsequent snapshots of the wave as it passes in front of the camera. Measuring the distance between the waves' crests in Fig. 2(b) and knowing the time between two snapshots (the imaging frequency rate), the wave speed can be deduced. The evolution of the speed with the number of laps is displayed in Fig. 3. This figure indicates that the wave speed has five distinct, almost constant, levels and jumps from one level to another. This behavior could be accounted for by the residual

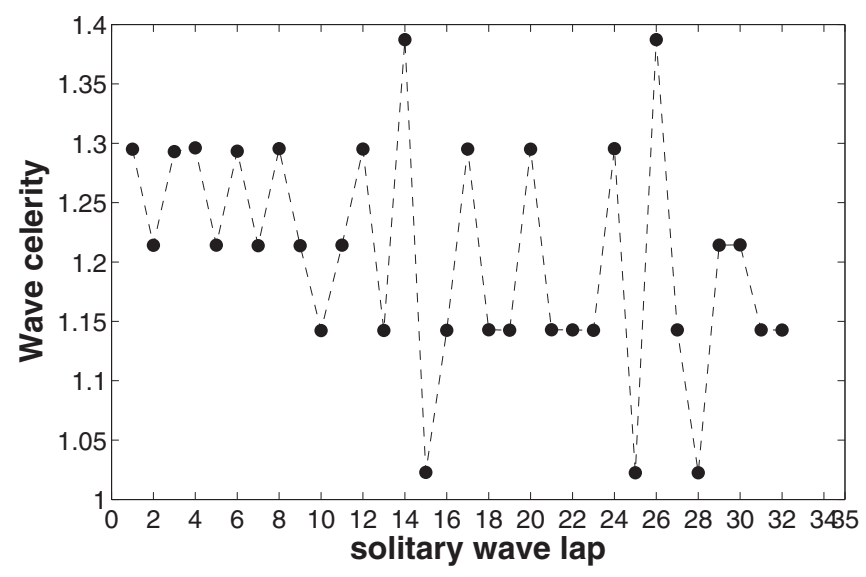

FIG. 3. Wave speed in the laboratory frame of reference versus number of laps. The water depth was $H_{0}=25.5 \mathrm{~mm}$. sloshing of the fluid, which occurs when swirling fluid is drained through a sink. The intrinsic wave speed in the rotating frame of reference was also not experimentally accessible. However, it can be estimated using the simplified theory of Ref. [11] as $\sqrt{g H_{0}}\left(1+\frac{1}{2} \frac{A}{H_{0}}\right)$. Based on these two wave speeds (absolute and relative) the rotational speed of the background flow, $\Omega_{0}$, can be estimated. Inserting the estimated value of $\Omega_{0}$ in the expression for $\alpha_{0}$ in Eq. (44), one can obtain the profile of the solitary wave at different stages (laps) of its development. Thus, the difference between the actual and the estimate values of $\Omega_{0}$ should affect the profile and the speed of the wave, deduced from the theory.

This influence should be more noticeable at the last stages of the lifetime of the wave, when the background rotation has decreased appreciably due to the accumulation with time of viscous effects. The typical Reynolds $\left(\operatorname{Re}=\frac{\Omega_{0} R^{2}}{v}\right)$ and Ekman $\left(\mathrm{Ek}=\frac{v}{\left(\Omega_{0} H^{2}\right)}\right)$ numbers based on the average estimated value of the background rotation are approximately $6 \times 10^{4}$ and $5 \times$ $10^{-4}$, respectively. Because of viscous effects, the actual value of $\Omega_{0}$ should be inferior and thus the values of Reynolds and Ekman numbers can be considered as moderate, specifically during the last laps of the wave, when the rotational speed of the background flow should have decreased enough that ignoring viscous effects becomes hard to justify. The Rossby number in our experiment, defined as Ro $=\frac{U}{2 R \Omega_{0}}$, is constant and equal to $1 / 2$ with $U=\Omega_{0} R$; this value also is moderate which suggests that the wave propagation is moderately influenced by the Coriolis force.

A comparison between the predicted wave profile (44) and that observed at different phases of the wave development is shown in Fig. 4. An assessment of the wave speeds (experimental and theoretical) is given in Table I. The first observation that one can infer is that the wave profile predicted by the theory is close to the one observed in the experiment but it is sharper than the actual profile. This difference might be explained by the presence of friction on the walls, which tends to stretch or enlarge the wave profile. The second observation is that the theory predicts the wave speed with good accuracy; see Table I. Figures 4(a)-4(c) compare the experimental and theoretical profiles in the time interval during which the wave was generated and traveled half the distance before it vanishes; i.e., between the first and the 15th laps. In this time interval, 

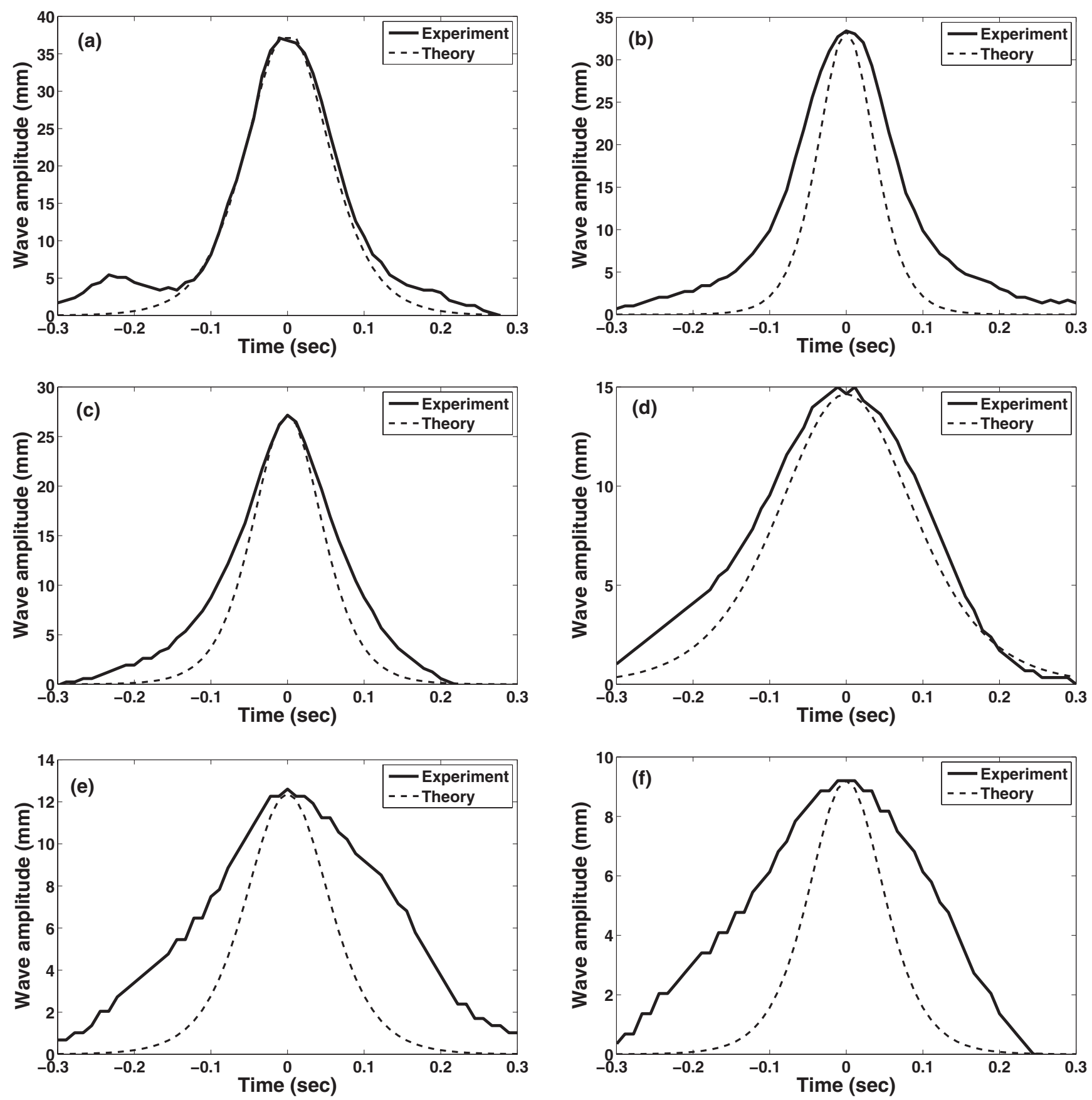

FIG. 4. Comparison between theoretical and observed wave profiles at the same space position after various laps, starting from the initial stage of the wave formation, after (a) one, (b) three, (c) seven, (d) 15, (e) 19, and (f) 24 laps. The experimental conditions were as follows: constant water depth $H_{0}=25.5 \mathrm{~mm}$, initial swirl $\omega=120 \mathrm{rpm}$, and initial water height $h=435 \mathrm{~mm}$.

given that the theory considers the fluid to be inviscid while water is viscous, the comparison between the wave profiles is fair. In the second half of the wave's lifetime the divergence between the two profiles becomes more significant. The reason behind this behavior is of course the viscous effects. In addition to the shear at the walls of the container, the accumulation with time of viscous influences became significant, which might result in large differences between the estimated and actual values of $\Omega_{0}$. The actual value of the background flow rotational speed should be much inferior to the one estimated using the inviscid theory [11]. Therefore, based on the fact that the rotational character of the background flow is reflected in both nonlinear and dispersion terms in Eq. (42), any difference in the value of $\Omega_{0}$ should affect the interplay between dispersion and nonlinearity during the wave propagation. For instance, an error of $10 \%$ in the wave speed in the laboratory frame of reference can significantly affect the shape of the theoretical profile, thus giving a reason for its deviation from the experimental profile. Surprisingly enough, the predicted wave speed remains close to the measured value; see Table I.

From this discussion it seems that that the viscosity affects the dispersion more than the nonlinearity of the wave. The 
TABLE I. Comparison between the wave speed predicted by the theory and that measured in the laboratory after different laps.

\begin{tabular}{lccc}
\hline \hline & $c($ expt $)(\mathrm{m} / \mathrm{s})$ & $c$ (theor) $(\mathrm{m} / \mathrm{s})$ & Error $(\%)$ \\
\hline Lap 1 & 1.2 & 1.29 & 7.2 \\
Lap 3 & 1.3 & 1.2 & 7 \\
Lap 7 & 1.1 & 1 & 1 \\
Lap 15 & 1.25 & 1.21 & 3.6 \\
Lap 19 & 1.12 & 1.14 & 1.5 \\
Lap 24 & 1.12 & 1.14 & 2 \\
\hline \hline
\end{tabular}

value of $\alpha_{0}$ that encompasses the influence of the rotational character of the background flow was varied approximately by $7 \%$ during the experiment. This variation alone seems not to greatly affect the nonlinearity of the wave, and hence its speed; see Eqs. (42) and (44). However, the relatively small variation of $\alpha_{0}$ combined with the dispersion of the wave appears to influence the wave propagation significantly, which might explain also why the wave became wider as it propagated. The frictional effects, which could not be captured by the inviscid theory, seem to play a role not only in modifying the shape and the speed of the wave on the cylindrical walls but also in affecting its dispersion through the background rotational speed. In fact, the width of the wave is larger in the experiments than the predictions.

\section{CONCLUSION}

In this paper, a KdV-type equation was derived, which governs a rotating solitary surface wave observed on the lateral wall during drainage of a water-filled cylindrical container under shallow water conditions. It represents an improvement of our previous work [11] where the radial component of the perturbed velocity field was neglected. The primary drawback of the earlier strong assumption produced the classical planar $\mathrm{KdV}$ equation which did not account for the rotational character of the base flow field. Since our interest was focused on the wall wave motion, a projection of the flow onto the lateral wall was considered. A less stringent approximation than a zero radial component at the wall is assumed, consisting in neglecting the radial curvature of the radial velocity at the wall. This approach might be justifiable since it preserves the three-dimensional character of the flow via the wall radial gradients of all the dependent variables. A KdV-type equation with coefficients different from those of the planar case was then obtained. As was expected, this formulation contained the rotational aspect of the base flow through the dependence of the equation coefficients on the background angular velocity. The theory was shown to predict with fair accuracy the shape and the kinematics of the observed wave. The frictional effects seem to play a role by modifying the shape and the speed of the wave at the cylindrical wall and increasing its dispersion through decrease of background rotational speed. A natural extension of the present work would be the inclusion of the viscosity in the analysis. Moreover, if improvements to the experimental apparatus are made, such as controlling the speed of the background flow or/and rotating the sidewalls to limit the shear stress, the modified experimental setup could serve as a tool to investigate the role of rotation in the propagation of solitary waves. Another natural extension of the present work, which is in progress, involves the derivation of a formulation applicable for the entire wave motion. However, the latter will certainly require a combination of both analytical and numerical treatments.

\section{ACKNOWLEDGMENT}

We thank an anonymous reviewer for the suggestion of rotating the sidewalls to limit the shear stress.

\section{APPENDIX}

Looking for a solution of Eqs. (15)-(17) in the traveling wave form, i.e., setting $(u, v, \eta)=(\tilde{u}(r), \tilde{v}(r), \tilde{\eta}(r)) \exp i k[\theta-$ $\left.\left(\Omega+\frac{1}{\alpha}\right) t\right]$ amounts to setting $D_{t}=-\frac{1}{\alpha} \partial_{\theta}$. Then the eigenvalue problem for the wave amplitude reads

$$
\begin{gathered}
-i \frac{k}{\alpha} \tilde{v}+2 \Omega \tilde{u}+i \frac{k}{r} \tilde{\eta}=0, \\
-i \frac{k}{\alpha} \tilde{u}-2 \Omega \tilde{v}+\tilde{\eta}_{r}=0, \\
-i \frac{k}{\alpha} \frac{r}{h_{0}} \tilde{\eta}+i k \tilde{v}+\tilde{u}+r \tilde{u}_{r}=0 .
\end{gathered}
$$

By eliminating $\tilde{u}, \tilde{v}$, and their radial derivatives in favor of $\tilde{\eta}$, the following eigenvalue equation for the wave amplitude modes is obtained:

$$
r^{2} \tilde{\eta}_{r r}+r \tilde{\eta}_{r}+\left\{4 \Omega^{2}-k^{2}\left(1-\frac{r^{2}}{\alpha^{2} h_{0}}\right)\right\} \tilde{\eta}=0 .
$$

This equation differs slightly from the Bessel equation due to the presence of $h_{0}(r)$. The boundary condition $\left.\tilde{u}\right|_{r=1}=0$ serves to determine the dispersion curve between the wave number $k$ and the wave speed $\Omega+\frac{1}{\alpha}$.
[1] E. Falcon, C. Laroche, and S. Fauve, Phys. Rev. Lett. 89, 204501 (2002).

[2] M. Peyrard and T. Dauxois, Physics of Solitons (Cambridge University Press, Cambridge, England, 2006).

[3] R. Grimshaw, Solitary Waves in Fluids (WIT Press, Southampton, UK, 2007).

[4] W. K. Melville, G. G. Tomasson, and D. P. Renouard, J. Fluid Mech. 206, 1 (1989).

[5] C. Katsis and T. Akylas, Phys. Fluids. 30, 297 (1987).

[6] R. Grimshaw, Stud. Appl. Math. 73, 33 (1985).

[7] W. G. Pritchard, J. Fluid Mech. 42, 61 (1970).
[8] E. Hopfinger and F. Browand, Nature (London) 295, 393 (1982)

[9] E. H. T. Maxworthy and K. Redekopp, J. Fluid Mech. 151, 141 (1985).

[10] G. H. Vatistas, J. Fluid Mech. 217, 241 (1990).

[11] H. A. Abderrahmane, M. Amaouche, M. Fayed, H. D. Ng, G. H. Vatistas, and K. Siddiqui, Phys. Rev. E 84, 037302 (2011).

[12] K. Helfrich, Phys. Fluids 19, 026601 (2007).

[13] R. Grimshaw and K. Helfrich, Stud. Appl. Math. 121, 71 (2008).

[14] T. Foglizzo, F. Masset, J. Guilet, and G. Durand, Phys. Rev. Lett. 108, 051103 (2012). 